\title{
Computed Tomography Findings of a Case of Megacolon Associated with Caudal Regression Syndrome
}

\author{
Fatih Celikyay ${ }^{\mathrm{a}}$, Ruken Yuksekkayaa, ${ }^{\mathrm{a},}$, Gonca Erbas ${ }^{\mathrm{b}}$, Nuray Voyvoda ${ }^{\mathrm{c}}$
}

\begin{abstract}
Caudal regression syndrome is a congenital disease that affects the development of distal spinal segments and is reflected by abnormalities in the pelvis and genitourinary and gastrointestinal systems along with severe deformities in the extremities and spinal problems. Our goal was to report the computed tomography (CT) findings of a case of megacolon associated with caudal regression syndrome (CRS) that presented with constipation that had lasted for approximately 25 days.
\end{abstract}

Keywords: Caudal regression syndrome; Megacolon; Computed tomography

\section{Introduction}

Caudal regression syndrome (CRS) is a rare neural tube defect that affects the terminal spinal segments [1]. It manifests as a partial agenesis of the thoracolumbosacral spine and is associated with genitourinary, gastrointestinal, and musculoskeletal problems $[1,2]$. Neurogenic bowel can give rise to incontinence, constipation or recurrent incontinence, and constipation attacks. Herein, we report on the computed tomography (CT) findings of a patient with a megacolon associated with CRS.

\section{Case Report}

A 26-year-old man was admitted to the gastroenterology

Manuscript accepted for publication May 16, 2013

${ }^{a}$ Radiology Department, Gaziosmanpasa University School of Medicine, Turkey

${ }^{\mathrm{b}}$ Radiology Department, Gazi University School of Medicine, Turkey

${ }^{c}$ Radiology Department, Acibadem Kocaeli Hospital, Turkey

${ }^{\mathrm{d} C}$ Corresponding author: Ruken Yuksekkaya, Radiology Department, Gaziosmanpasa University School of Medicine, Turkey.

Email: rukenyuksekkaya@yahoo.com

doi: http://dx.doi.org/10.4021/jmc1321w department with abdominal distension and constipation that had lasted for approximately 25 days. The patient's medical history revealed that he had been diagnosed with type I diabetes and hypertension and had complained of urinary and chronic refractory defecation. His mother was also diagnosed with type I diabetes, and his sister not only had this disease, but was also diagnosed with sacral agenesis. On physical examination, his abdomen was markedly distended. In addition, no bowel sounds were heard, and no fever or other toxic symptoms were observed. Moreover, routine laboratory tests indicated nothing out of the ordinary.

An abdominal CT examination with oral and intravenous contrast material showed that the rectum and sigmoid colon were significantly dilated with feces and gas, but no haustration was observed in the sigmoid colon. Furthermore, there was no thickening or contrast enhancement in the bowel wall after the administration of the contrast material. Bilateral moderate hydronephrosis and ureteral dilatation were also revealed. The loops of the small bowel were filled normally with the oral contrast material, and because of the bulky, dilated sigmoid colon and rectum, they were displaced to the

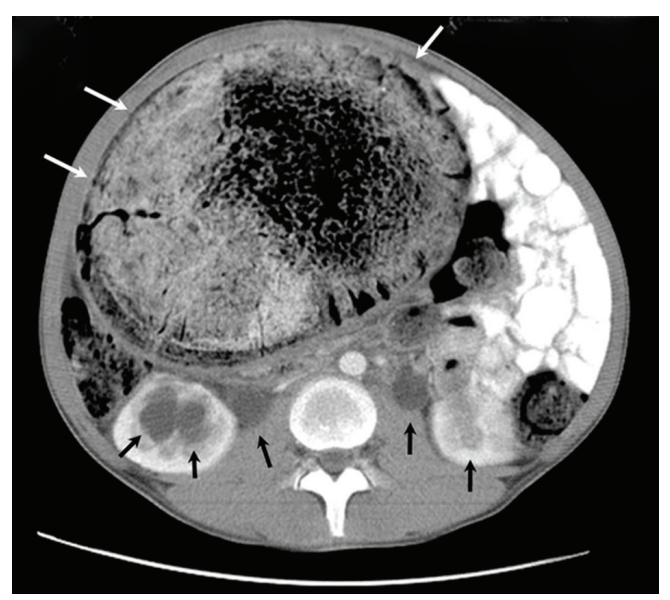

Figure 1. An axial CT scan shows the huge, dilated, nonhaustrated sigmoid colon (white arrows), bilateral dilated urinary calices, and ureters (black arrows). The thickness of the bowel wall is normal, and there is no contrast enhancement. The compressed and displaced small bowel on the left side of the abdomen can also be seen. 

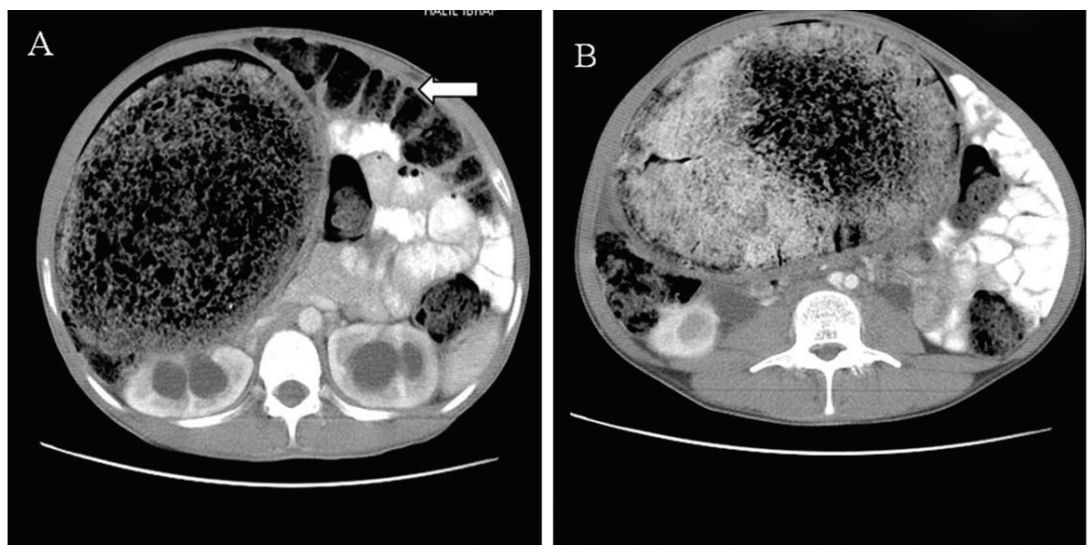

Figure 2. A, B. Axial CT scans show the huge, dilated, non-haustrated sigmoid colon. The loops of the large bowel are also dilated and filled with feces. They are not filled with oral contrast material, and their normal haustration pattern is preserved. The compressed and displaced small bowel on the left side of the abdomen is also shown.

left side of the abdomen (Fig. 1). However, the loops of the other parts of the large bowel were not filled with the oral contrast material but were dilated and filled with feces and air. Their normal haustration pattern was preserved (Fig. 1, 2). Additionally, the patient's bladder was displaced anteriorly out of the pelvis and was thickened diffusely and irregularly at the left lateral side (Fig. 3). The coccyx and distal portion of the sacrum below the level of the sacral second vertebra were also absent (Fig. 4). These findings supported the diagnosis of type II sacral agenesis. The patient was then treated symptomatically, discharged with laxatives, and given appropriate advice regarding his care.

\section{Discussion}

The incidence of CRS is estimated at approximately one in

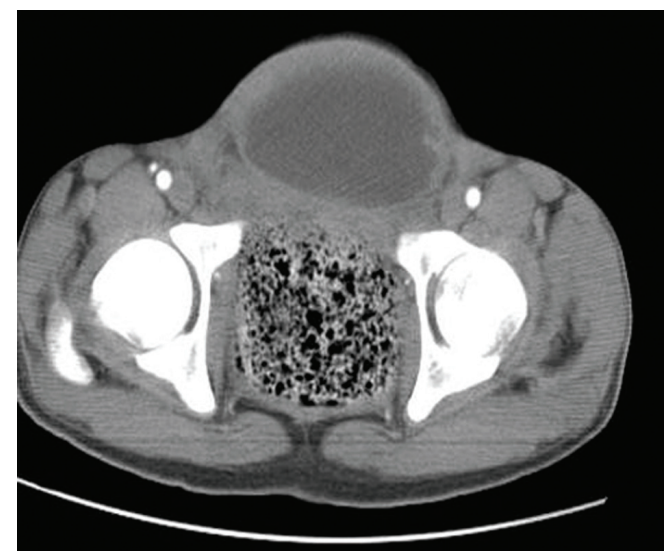

Figure 3. An axial CT scan shows the anteriorly displaced bladder with irregular wall thickening (black arrows) along with the absence of inferior sacrococcygeal vertebrae (white arrows). every 60,000 births with a male to female ratio of 2.7 to 1 $[1,3]$. Musculoskeletal and genitourinary abnormalities can be associated with this disease as well as gastrointestinal abnormalities such as neurogenic bowel, an imperforate anus, rectovaginal fistulas, omphaloceles, Meckel's diverticulum, and massive rectal prolapses. Neurogenic bowel can give rise to incontinence, constipation or recurrent incontinence, and constipation attacks.

The exact etiology and pathogenesis of CRS are not known and incidentally diagnosed asymptomatic cases have also been reported in the literature [4]. However maternal diabetes, genetic predisposition, trauma, toxic agents, vascular hypoperfusion, and nutritional problems have been suggested in etiology $[2,5]$. The incidence of sacral agenesis in babies of diabetic mothers is $1 \%$, and the incidence of maternal diabetes in patients with sacral agenesis is about $16-20 \%$ [6]. In our patient, there was a history of maternal diabetes,

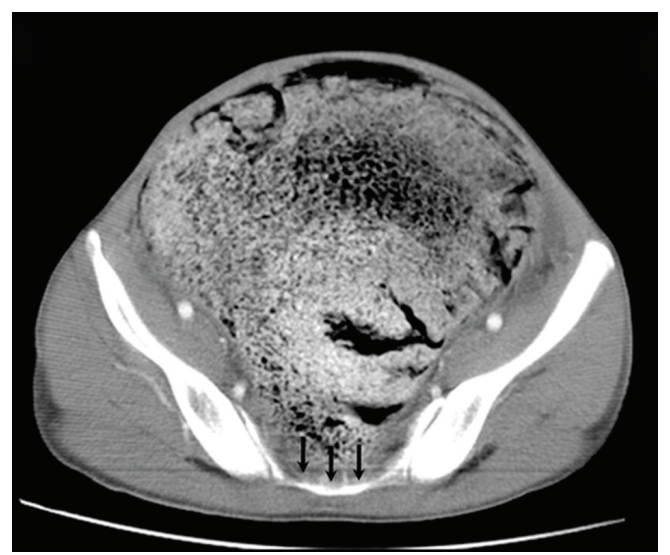

Figure 4. An axial CT scan shows the absence of inferior sacral vertebrae (black arrows). Abdominal distension secondary to the huge, dilated bowel which fills the whole pelvis is also shown. 
and he also had type I diabetes.

Renshaw has classified sacral agenesis into four types. In type I, there is either partial or total unilateral sacral agenesis. Type II consists of partial sacral agenesis with a partial but bilateral symmetrical defect and stable articulation between the iliac bones and remaining sacrum. Type III features variable lumbar and total sacral agenesis, with the ilia articulating with the lowest vertebra present. Type IV has variable lumbar or total sacral agenesis, with the caudal endplate of the lowest vertebra resting above either fused ilia or an iliac amphiarthrosis [7]. According to this classification system, our patient had type II sacral agenesis. Therefore, the first and second sacral segments were present, and there was a normal articulation between these segments and the iliac bones.

The differential diagnoses of this case included megacolon and toxic megacolon, a life-threatening complication of other intestinal conditions that causes rapid dilatation of the colon within one to a few days. The symptoms of toxic megacolon are abdominal pain, distension, tenderness, fever, and a rapid heart rate. In some cases, there is even shock [8]. However, megacolon does not cause sudden, life-threatening toxic symptoms. Our patient had a history of abdominal distension that had lasted for approximately 25 days and a history which indicated this had been an ongoing complaint since birth. However, he had no toxic symptoms to indicate the presence of toxic megacolon. Megacolon can be caused by Hirschsprung's disease (HD), bowel muscle paralysis, hypothyroidism, laxative overuse, avoidance of defecation (due to depression, dementia, schizophrenia, or anorectal pain), familial infantile metachromatic leukodystrophy, colonic pseudo-obstruction, familial visceral myopathy, systemic sclerosis, Chagas' disease, adrenomyodystrophy, or constipation [9].

The sigmoid colon and rectum are innervated by the inferior hypogastric plexus, consisting of the hypogastric nerve and the pelvic splachnic nerves. These nerves, originate from the sacral third and fourth segments, then innervate the left colon, sigmoid colon, rectum, and anus [10]. In our patient, there were no sacral third and fourth segments, so the innervation of the sigmoid and rectum was defective, and this probably gave rise to the development of the megacolon. The kidneys and ureters are innervated by the thoracal and lumbal segments; therefore, the hydronephrosis and dilatation of the ureters in our patient occurred because of the compression of the significantly dilated rectum and sigmoid colon on the ureters.

Computed tomography is usually accepted as the primary method for evaluating colonic diseases since it is widely available and easily performed. In addition, CT provides the measurement of bowel diameter and bowel wall thickness, indicates intramural disease, and shows the pericolonic soft tissues, neoplasms, abdominal organs, and bony structures. On CT, a normal colon measures up to $9 \mathrm{~cm}$ at the cecum, with the other segments being shorter. The bowel wall thickness is $1-2 \mathrm{~mm}$ when distended and $3-4 \mathrm{~mm}$ when collapsed [11]. Megacolon is the term used when the diameters of the cecum, rectosigmoid region, and ascending colon are greater than $12,6.5$, and $8 \mathrm{~cm}$, respectively [12]. Our patient had a huge rectum, and the sigmoid colon filling the abdomen measured greater than $12 \mathrm{~cm}$.

Toxic megacolon barium studies are better than $\mathrm{CT}$ for evaluating superficial changes, but CT can show the dilated segments, level of obstruction, and the thickened, layered and contrast-enhanced bowel walls along with complications such as perforation, pneumatosis, and abscess [11]. In HD, radiographs of the abdomen show massive distension of the proximal segments of the colon along with a small, narrowed distal segment, whereas single-contrast barium enema examinations reveal highly dilated segments filled with feces and cone- or funnel-shaped transition segments proximal to the narrow segment [13]. Computed tomography can also show the dilated segment, transition zone, and narrowed distal segment without any other obstructive cause. We did not diagnose our patient as having HD because there were no dilated and narrowed segments. Dilation did occur, especially in the rectum and colon, but the other parts of the colon were also dilated. To our knowledge, there have been no other case reports like ours in which the CT findings indicate a diagnosis of megacolon associated with CRS.

In conclusion, CRS must be considered in the differential diagnosis of megacolon since it is a rare cause of both constipation and colonic distension. Computed tomography can be utilized in the evaluation of megacolon and can also be used to differentiate megacolon from toxic megacolon by showing the abnormalities of the other abdominal organs and bony structures which can provide clues regarding the etiology.

\section{Conflicts of Interests}

The authors declared no conflicts of interests. The work has been performed Gazi University School of Medicine Radiology Department.

\section{References}

1. Singh SK, Singh RD, Sharma A. Caudal regression syndrome--case report and review of literature. Pediatr Surg Int. 2005;21(7):578-581.

2. Kahilogullari G, Tuna H, Aydin Z, Vural A, Attar A, Deda H. Caudal regression syndrome diagnosed after childhood period: a case report. Neuroanatomy. 2005;4:17-18.

3. Stevenson RE, Jones KL, Phelan MC, Jones MC, Barr M, Jr., Clericuzio C, Harley RA, et al. Vascular steal: 
the pathogenetic mechanism producing sirenomelia and associated defects of the viscera and soft tissues. Pediatrics. 1986;78(3):451-457.

4. Tunell WP, Austin JC, Barnes PD, Reynolds A. Neuroradiologic evaluation of sacral abnormalities in imperforate anus complex. J Pediatr Surg. 1987;22(1):58-61.

5. Duhamel B. From the Mermaid to Anal Imperforation: The Syndrome of Caudal Regression. Arch Dis Child. 1961;36(186):152-155.

6. Guzman L, Bauer SB, Hallett M, Khoshbin S, Colodny $\mathrm{AH}$, Retik AB. Evaluation and management of children with sacral agenesis. Urology. 1983;22(5):506-510.

7. Padmanabhan R. Retinoic acid-induced caudal regression syndrome in the mouse fetus. Reprod Toxicol.
1998;12(2):139-151.

8. http://www.nlm.nih.gov/medlineplus/ency/article/0248. htm.

9. http://radiozen.wordpress.com/28/7/16/gi-differentialdiagnoses/.

10. Snell RS. Autonomous Nervous System. In: Snell RS ed. Clinical Anatomy for Medical Students. Nobel Turkish Edition 1995;464-476.

11. Thoeni RF, Cello JP. CT imaging of colitis. Radiology. 2006;240(3):623-638.

12. Koeberle F. Enteromegaly and Cardiomegaly in Chagas Disease. Gut. 1963;4:399-405.

13. Mindelzun RE, Hicks SM. Adult Hirschprung disease: radiographic findings. Radiology. 1986;160(3):623-625. 